CORRIGENDUM

\title{
Haemangiopericytoma of the hard palate
}

A Ceylan, K Degerliyurt, F Çelenk, MS Ataç and S Sabri Uslu

Dentomaxillofacial Radiology (2010) 39, 454. doi: 10.1259/dmfr/28330117

Correction to: Dentomaxillofacial Radiology (2008) 37, 58-61. doi: 10.1259/dmfr/65740001

The authors regret that in the above paper the second author name was given incorrectly. The correct author name is: K Degerliyurt. 